\title{
'Oral health matters': it is time for a culture change in dentistry
}

Peter Mossey ${ }^{1}$

\section{Key points}

Dentistry sits at a crossroads where future paths include ongoing intervention for oral disease or a much greater focus on primary prevention.
What is needed is a culture change underpinned by collective responsibility, individual behaviour change and new policies with a range of upstream and downstream actions.
In 2020, leadership within the dental profession and unique circumstances means we are equipped to take advantage of unprecedented opportunities to drive the primary prevention agenda.

\begin{abstract}
This opinion is borne out of my involvement in and experience with colleagues at the International Association for Dental Research (IADR), the International Dental Federation (FDI) and the World Health Organisation (WHO), and seeks to provide a strong rationale for the dental profession embracing the advantages of a primary preventive agenda. Five key actions are used to emphasise our role in health and wellbeing, addressing inequalities, engaging more with other healthcare colleagues, being trans-sectoral (beyond health) in our approach and being prepared to embrace change. The thrust of the argument is that dentistry has been significantly undervalued in the healthcare field in the past, and can play a unique and leading role in future health and wellbeing through primary prevention.
\end{abstract}

The headline statement of volume 394, number 10194 of The Lancet in July 2019 was: 'Oral health matters. The teeth and mouth are an integral part of the body, supporting and enabling essential human functions, and the mouth is a fundamental feature of personal identity?. This issue attracted a global audience as it was launched in London in August 2019 and was seen as a renewed offensive to ensure the place of oral health in general health is acknowledged globally.

I see this as part of a wider global initiative involving the International Dental Federation (FDI), the WHO Oral Health and the International Association for Dental Research (IADR) to bring oral health to the top table and to attract the serious attention of health ministers and governments around the world to address this. The editor of The Lancet, Richard Horton, in the summing up at that event challenged us in dentistry to muster more power within the arguments, more 'anger' (in Professor Horton's words) and

'School of Dentistry, University of Dundee, UK. Correspondence to: Peter Mossey

Email address: p.a.mossey@dundee.ac.uk

Accepted 12 March 2020

https://doi.org/10.1038/s41415-020-1634-3 greater demonstration of resolve to achieve a different outcome. Below, I will outline why oral health deserves greater priority and why now is an ideal time for action, and I will attempt to suggest five ways where this might be achieved.

\section{Action 1}

We must expand our actions in addressing inequalities and injustice, and while there is a social gradient in every country in the world and this is sensitively reflected in oral health, we must take much more cognisance of the lowest-income countries and marginalised and vulnerable groups, and target oral diseases beyond dental caries and periodontal disease. In the context of access to care, countries in South East Asia and Sub-Saharan Africa have an extreme deficit in their dental workforce, expertise and affordability of basic care. Many Sub-Saharan African countries were unable to meet the Millennium Development Goals in basic issues such as clean water, sanitation, child mortality and maternal mortality. An oral condition called cancrum oris (or noma), a devastating and disfiguring gangrenous destruction of oral and facial tissues, only occurs in the world's most deprived communities, and in such deprived communities the vast majority of infants born with cleft lip and palate do not survive the first few weeks of life. ${ }^{1}$ The leadership of the dental profession has an absolute right to be angry about such stark inequities, injustices and denial of the basic human right to have access to healthcare, and it is incumbent on us to address them.

\section{Action 2}

We must engage in a meaningful way with our colleagues in medicine and ensure that we build up mutual trust. Dental and oral diseases do not occur in isolation; they commonly occur alongside other non-communicable diseases (NCDs), such as cardiovascular disease, metabolic syndrome and type 2 diabetes. This is due to common risk factors such as diet, lifestyle (smoking/alcohol use), stress, hygiene and lack of exercise, and makes it much more cost-effective when introducing person-centred disease management strategies; for example, behaviour change whereby individuals are encouraged to take responsibility for their own health and wellbeing. We must also emphasise the value of the oral cavity as a window on the body; early 
detection of systemic disorders due to oral manifestations - such as ulcers, white patches, red patches, swellings, abnormal pigmentation and oral pain, loss of sensation, abnormal orofacial movement, halitosis and dry mouth - make a compelling case for oral health professionals working collaboratively with our medical colleagues in the context of screening and early detection of chronic diseases. The bidirectional relationship between periodontal disease and type 2 diabetes, the relationship between oral and cardiovascular disease and the growing trend for using saliva or other oral tissues as biomarkers for systemic disorders is now well established. The Non-Communicable Diseases Alliance (NCDA) provides the ideal forum for this conversation between the dental and medical professions, and with IADR and FDI both recently aligned to NCDA, we must engender mutual trust and cooperation between the medical and dental professions.

\section{Action 3}

We must speak to audiences and tell our stories outside of dentistry and outside of health; for example, to politicians and policymakers in countries all over the world. We remain largely introverted in our approach, and in many fora we are making very strong arguments on oral health, but often these are 'speaking to the converted. We must use the unique and unprecedented opportunity of the Minamata Convention as a tool for raising awareness of governments all over the world who have signed up to this convention and are expecting a response. On 24 December 2019, the government of Equatorial Guinea deposited its instrument of ratification, thereby becoming the 116th party to the Minamata Convention. In our response, the oral health profession can emphasise the importance of primary prevention as the best possible strategy to phase down use of dental amalgam and mercury contamination in the environment. This provides us with an opportunity for upstream advocacy to 116 governmental agencies to demand action on dental caries prevention through hygiene, fluoride and action on sugars and diet. There are already examples of successes in oral health improvement projects in various parts of the world, through high level advocacy and action (for example, Scotland, Portugal, Mexico) and initiatives in low- and middle-income countries (such as the new dental school in Malawi) these should be used as exemplary strategies that could be rolled out elsewhere.

\section{Action 4}

We must take advantage of a unique feature of oral health among NCDs, which is access to the healthy population that dentistry provides. In January 2020, a Scottish government report indicated that $95.7 \%$ of the population are registered with an NHS dentist, with similar rates for children and adults. This unique access to the healthy population across the life course provides the opportunity for lifestyle advice (for example, smoking cessation and exercise), screening (for example, for hypertension, blood sugars, cholesterol), and longitudinal monitoring as an adjunct to primary prevention and medical interventions for NCDs. We must therefore adopt a more holistic view of health (not confined to dental diseases), and forge strong alliances with the broader NCD group to make oral diseases a global public health priority - and through the Sustainable Development Goals agenda, a political priority across the world. Oral health must be seen as a valued partner in the NCDA through our IADR and FDI membership; this has already proven to be effective with oral health included in the September 2019 UN High-Level Meeting on Universal Health Coverage. We must also use oral cancer as an example of a silent killer that is on the increase in the younger population in many parts of the world. This relates to: a) tobacco cessation and alcohol consumption and risk, plus b) the issue of HPV vaccinations in both males and females - and the role of the dental profession in both of these issues is noteworthy.

\section{Action 5}

We must alter the current balance in dentistry between intervention and prevention, favouring the latter by integration of oral with general health and health with social care. The strategies for implementing this will require both upstream and downstream measures. We must stop talking about health alone and ensure that the message in the future is about health and wellbeing. To action this, we must strengthen the evidence base for oral health links with mental health, depression and quality of life. We must advocate upstream population-wide policy measures that seek to enhance awareness of social determinants and common risk factors for NCDs, including oral health-driven action on sugars (via legislation, regulation and taxation). Downstream, we must work with other health professionals to co-design strategies to integrate at all levels, with a combination of communitybased programmes carried out in schools, workplaces and communities to promote oral and general health and overall wellbeing, and person-centred healthcare services. This can be achieved using tools such as health coaching, capable of providing individualised care to people with (often comorbid) oral and general health conditions. This will require different and more flexible remuneration and workforce models, involving intra- and inter-professional trust and co-operation. The health economics of alternative modes of dental healthcare delivery in different countries, economic circumstances and cultures will be necessary to address the spiralling costs of dental care and out of pocket expenditure. The concept of universal health coverage (UHC) is based on availability, accessibility and affordability, and will be underpinned by appropriate workforce models, integration with general health in the drive towards prevention and a new era of university education and training, behaviour change and information dissemination.

\section{Conclusion}

These five key issues are of significant personal importance, in that my role as of Saturday 21 March 2020 is to undertake the Presidency of the IADR Global Oral Health Inequalities Research Network (GOHIRN) and to complement the work of many colleagues such as those in FDI and WHO Oral Health, and those who presented at The Lancet launch in London. This is important in that implementation of the actions will require a concerted effort with strategic actions via appropriate fora where authority, influence and leadership can be brought to bear, and where the sense of injustice represented by neglect of oral health (and indeed general health also) will resonate and gain support. We need a collective will and absolute unity within our three global bodies in dentistry FDI, WHO and IADR, whose strengths are complementary and synergistic and whose influence can be expressed via the NCDA. Achieving these actions will also require belief and internal motivation, with attitudinal and behavioural change; difficult to achieve and elusive in the past, but I believe possible with the help of tools now available in healthcare, a powerful example of which is health coaching.

\section{References}

1. Mossey P A, Modell B. Epidemiology of Oral Clefts 2012: An international perspective. In Cobourne M T (ed) Cleft Lip and Palate: Epidemiology, Aetiology and Treatment. pp 1-18. London: Karger Press, 2012. 